\title{
Desmoplasia and Detached Papillae in Early Esophageal Adenocarcinoma: A Histologic Study on Endoscopic Submucosal Dissection Specimens
}

\author{
Zhongbo Jin ${ }^{\mathrm{a}}$, Dongwei Zhang ${ }^{\mathrm{a}}$, Qin Zhang ${ }^{\mathrm{b}}$, Jinping Lai ${ }^{\mathrm{a}}$, Ashwin Akki ${ }^{\mathrm{a}}$, \\ Ashwini Esnakula ${ }^{a}$, Jesse Kresak ${ }^{a}$, David Hernandez Gonzalo ${ }^{a}$, \\ Peter V. Draganov ${ }^{\mathrm{c}}$, Hao Xie ${ }^{\mathrm{d}}$, Xiuli Liu ${ }^{\mathrm{a}, \mathrm{e}}$
}

\begin{abstract}
Background: Desmoplasia and detached papillae were only rarely mentioned in intramucosal adenocarcinoma of esophagus or stomach. This study aimed to examine these two features in early esophageal adenocarcinoma.

Methods: All endoscopic submucosal dissections specimens performed for Barrett's esophagus neoplasm during the year 2013 to 2016 were reviewed. These 44 cases included in this study were eight Barrett's esophagus with high-grade dysplasia, 21 intramucosal adenocarcinoma, and 15 submucosally or beyond invasive adenocarcinoma.
\end{abstract}

Results: Desmoplasia occurred in $73 \%$ submucosally or beyond invasive adenocarcinoma, higher than intramucosal adenocarcinoma $(24 \%)$ and high-grade dysplasia $(0 \%)$ ( $\mathrm{P}<0.00001$ for each). The frequency of detached papillae in intramucosal adenocarcinoma and submucosally or beyond invasive adenocarcinoma specimens was $71.4 \%$ and $73.3 \%$, higher than high-grade dysplasia $(0 \%, \mathrm{P}<0.0001$ for both). Univariate analysis identified desmoplasia as risk factors for lymphovascular invasion in intramucosal adenocarcinoma specimens (odds ratio $12, \mathrm{P}=0.048$ ), and desmoplasia and tumor thickness for lymphovascular invasion in intramucosal adenocarcinoma and submucosally or beyond invasive adenocarcinoma specimens combined (odds ratio 9.0, $\mathrm{P}=0.005$; odds ratio 2.7, $\mathrm{P}=0.01$, respectively). Age, gender, the largest dimension and the average thickness of endoscopic submucosal dissection specimens, tumor size, detached papillae, and poor differentiation were not associated with lympho-

Manuscript submitted February 3, 2019, accepted March 19, 2019

a Department of Pathology, Immunology and Laboratory Medicine, University of Florida, Gainesville, FL, USA

${ }^{b}$ Department of Pathology, The Third Central Hospital of Nankai University, Tianjin, China

'Division of Gastroenterology, Hepatology and Nutrition, Department of Medicine, University of Florida, Gainesville, FL, USA

${ }^{\mathrm{d} D i v i s i o n}$ of Hematology and Oncology, Mayo Clinic, Rochester, MN, USA ${ }^{e}$ Corresponding Author: Xiuli Liu, Department of Anatomic Pathology, Immunology and Laboratory Medicine, University of Florida, Gainesville, FL 32610, USA. Email: xiuliliu@ufl.edu

doi: https://doi.org/10.14740/gr1154 vascular invasion ( $\mathrm{P} \geq 0.05$ for all). Multivariate analysis confirmed that only desmoplasia was predictive of lymphovascular invasion (odds ratio 8.0, $\mathrm{P}=0.02$ ) in intramucosal adenocarcinoma and submucosally or beyond invasive adenocarcinoma specimens combined.

Conclusions: In conclusion, desmoplasia occurs in about a quarter of esophageal intramucosal adenocarcinomas and three quarters of submucosally or beyond invasive adenocarcinomas, and is associated with lymphovascular invasion in early esophageal adenocarcinoma.

Keywords: Barrett's esophagus; Intramucosal adenocarcinoma; Submucosally invasive adenocarcinoma; Desmoplasia; Lymphovascular invasion

\section{Introduction}

Advances in endoscopic therapy in the past decade have broadened the pool of patients with Barrett's esophagus (BE)-associated neoplasia for endoscopic intervention and diminished the need for esophagectomy in this patient population [1]. Esophagectomy should be reserved for patients with a long segment of intramucosal adenocarcinoma (IMAC) or those in whom endoscopic therapies fail or are inappropriate, such as the patients with a high risk of nodal metastases. In addition, recent research has suggested that early Barrett's carcinoma with "low-risk" submucosal invasion may be treated with endoscopic resection with a curative intent [2]. Thus, a correct diagnosis of early esophageal adenocarcinoma and risk stratification in this population using cancer characteristics obtained from endoscopic mucosal resection (EMR) and/or endoscopic submucosal dissection (ESD) specimens become critical in the subsequent management and treatment of these patients.

Histological diagnosis of IMAC and SMAC is challenging. IMAC is defined as a neoplasm that has invaded into the surrounding lamina propria or muscularis mucosae (MM) but not into the submucosa. Four criteria were used to establish a diagnosis of intramucosal adenocarcinoma: single cell invasion of the lamina propria in more than one focus; sheets of cells obliterating the lamina propria; small angulated, socalled abortive glands invading the lamina propria; or a never- 
ending/anastomosing gland pattern similar to endometrial adenocarcinomas of the uterine corpus [3]. It appears that there are two schools of thoughts regarding the diagnosis of SMAC; one requires the presence of unequivocal or "textbook worthy" stromal desmoplasia [3]; and the other one depends on the infiltration of neoplastic cells into the submucosa [4]. Although desmoplastic response to tumor was reported in areas of tumor infiltration into the duplicated MM space [5], the frequency and significance of this feature in early esophageal adenocarcinoma have not been examined.

The aims of this study were to determine: 1) The frequency of stromal changes and detached papillae in early esophageal adenocarcinoma in ESD specimens; 2) The association of these two features with lymphovascular invasion (LVI).

\section{Materials and Methods}

\section{Study population}

Approval was obtained from the Institutional Review Board at University of Florida, Gainesville, FL. The study population comprised 44 patients who had undergone ESD at the Shands Hospital Barrett's Esophagus Unit from 2013 to 2016.

\section{Clinicodemographics of the ESD cohort}

Data were collected on demographic and clinical and endoscopic variables using medical charts, including age at the time of ESD procedure, gender, the presence of BE mucosal irregularities, i.e., mucosal ulceration, nodules and plaque-like lesions. Biopsy results prior to ESD were also recorded.

\section{ESD technique and specimen preparation}

All procedure was performed under general anesthesia by a single endoscopist (P.V.D.) as previously reported [6]. All ESD specimens were pinned out flat on a cork or foam board and fixed in 10\% neutral buffered formalin for overnight. The largest size and average thickness of the ESD specimen of all cases was measured after fixation and recorded in the macroscopic description. All specimens were examined for the presence of mucosal lesions. All peripheral and deep margins were inked and submitted using the protocol as previously reported [7]. All specimens were entirely submitted for histology using a 2-mm interval between sections. The tissue were processed routinely, embedded in paraffin and sectioned at $5-\mu \mathrm{m}$ thickness for hematoxylin-eosin (H\&E) staining.

\section{Pathology review and histologic evaluation}

All pathology reports were reviewed. Information on the largest dimension of ESD specimen and average thickness of ESD was obtained. Information on the gross features, i.e., mucosal ulceration, nodules, and plaque-like lesions, was derived from the original pathological report. In addition, all slides were reviewed by one gastrointestinal pathologist (X.L.) to confirm the diagnosis, measure tumor thickness and tumor size, margin status, and assess the presence of LVI, stromal changes, and detached papillae. The diagnosis of IMAC was based on the criteria described previously [3]. Multifocality of IMAC was defined as the presence of $\geq$ two discrete IMACs separated by non-carcinomatous mucosa either grossly or microscopically after reviewing and matching the pathological reports and corresponding slides. The diagnosis of SMAC was based on infiltration of neoplastic cells into the submucosa [4]. Submucosa is defined as loose connective tissue containing area below a thickened double MM [4], space with mucus glands, or space with large-caliber arteries. The tumor grade was assigned according to the definitions of the American Joint Committee on Cancer: G1, well-differentiated; G2, moderately differentiated; G3, poorly differentiated; G4, undifferentiated. Tumor thickness was measured microscopically from the surface of the tumor to the area of the deepest invasion. In cases with multifocal IMAC, the thickness of the thickest lesion was measured and used for the entire case. LVI is defined as the presence of tumor emboli in endothelial cell-lined spaces in the invasive front of the tumors [8]. Cases that had clusters of tumor cells immediately adjacent to endothelial cell-lined spaces or tumor cells in empty spaces without endothelial lining were considered possible LVI and were subjected to immunohistochemistry for D240, ERG, and/or CD31. In addition, desmoplasia, defined as the presence of activated fibroblasts and myofibroblasts with extracellular matrix deposition (Fig. 1a) was evaluated. Cases with only myxoid stromal change (Fig. 1b) were considered desmoplasia-negative. Further, the presence or absence of detached papillae, defined as clusters of tumor cells without attachment to the surface or luminal epithelium (Fig. 1c, d), was recorded.

\section{Statistical analysis}

Descriptive statistics were computed for all variables. These included means and standard deviations (SD) for continuous factors and frequencies for categorical factors. Comparisons between two groups were made by using the two-tailed $t$ test (or Wilcoxon rank sum test as appropriate) for continuous variables and Chi-square test (or the Fisher exact test as appropriate) for categorical variables. Logistic regression was used in univariate and multivariable analysis. Variables with $\mathrm{P}<0.1$ from univariate analysis were included in multivariable analysis. Statistical analysis was performed with the SPSS software version 16 (SPSS, Chicago, IL). P value $<0.05$ is considered statistically significant.

\section{Results}

\section{Clinicodemographics of the ESD cohort}

A total of 44 cases were included in this study. Forty (91\%) were male and four ( $9 \%$ ) female; and the mean age at the time of ESD procedure was 65.9 (SD: 9.2) years. Among them, 40 


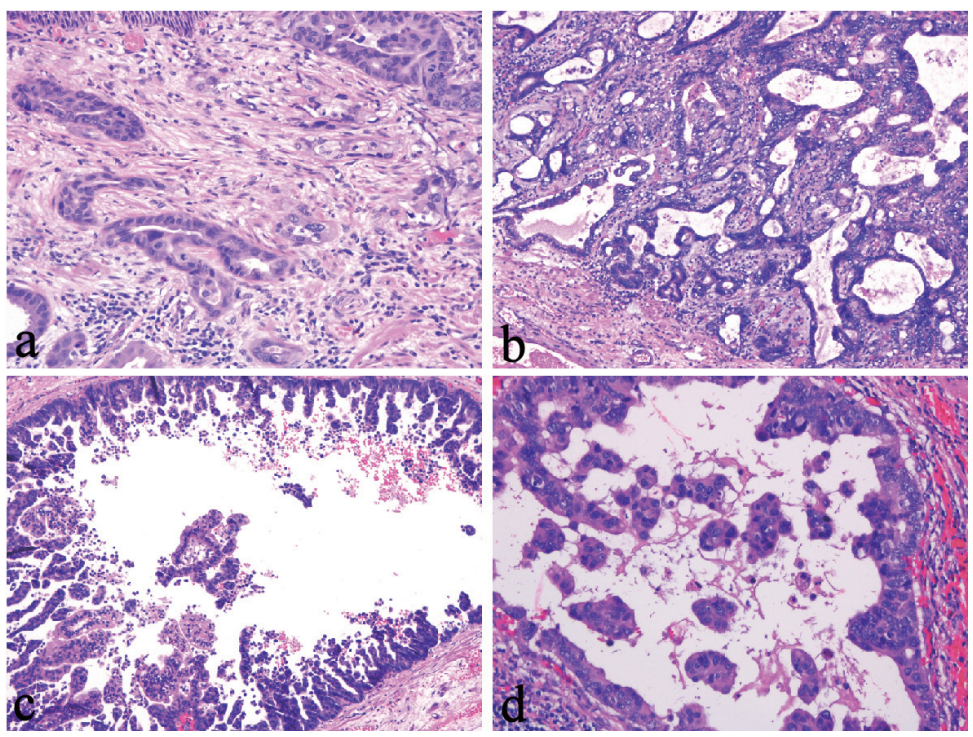

Figure 1. (a) Desmoplasia in an esophageal intramucosal adenocarcinoma (IMAC) $(400 \times)$. (b) Myxoid stromal changes in an esophageal IMAC $(100 \times)$. (c, d) Detached papillae in an esophageal IMAC $(100 \times$ and $200 \times)$.

(90.9\%) had endoscopically evident esophageal nodule, one had plaque $(2.3 \%)$, and three $(6.8 \%)$ had no obvious lesion. Thirty-seven $(84.1 \%)$ cases had ESD performed for biopsy proven HGD $(\mathrm{n}=17)$ or higher (IMAC $(\mathrm{n}=19)$ and SMAC $(\mathrm{n}=1))$, one $(2.3 \%)$ for LGD, four $(9.0 \%)$ with unspecified diagnoses and the remaining two cases $(4.6 \%)$ for esophageal nodule without previous biopsy.

En bloc resection was achieved in all cases. The ESD specimens had an average thickness of $2.92 \mathrm{~mm}$ (SD: 0.22). Thirty-three $(75 \%)$ had lesions on macroscopic examination including 18 with nodules, seven plaque, six ulcerated lesion, one granularity, and one retraction.

These included eight cases with Barrett's esophagus with HGD (BE-HGD), 21 IMAC, and 15 invading the submucosal or beyond (SMAC). Seven cases (15.9\%) including two IMAC and five SMAC had positive deep margin. Four (out of 36, $11.1 \%$ ) cases had multifocal adenocarcinoma. The average size of the largest carcinoma was $10.2 \mathrm{~mm}$ (SD: $6.4 \mathrm{~mm})$. The average thickness of carcinoma was $1.98 \mathrm{~mm}$ (SD: $1.47 \mathrm{~mm}$ ). Seventeen cases (out of 36, 38.6\%) had LVI. Examples of BEHGD, IMAC, and SMAC are illustrated in Figure 2.
Frequency of stromal changes in superficially invasive esophageal adenocarcinoma in ESD specimens

The frequency of desmoplasia in SMAC specimens was 73\%, higher than IMAC (24\%) and BE-HGD $(0 \%)(\mathrm{P}<0.00001$ for each). A positive association of desmoplasia and the presence of LVI was noted; $80 \%$ with desmoplasia were positive for LVI vs. $25 \%$ without desmoplasia $(\mathrm{P}=0.048)$ in IMAC specimens and $75 \%$ vs. $25 \%(\mathrm{P}=0.006)$ in IMAC and SMAC specimens combined (Table 1). In SMAC specimens, 73\% with desmoplasia were positive for LVI vs. $25 \%$ with no desmoplasia $(\mathrm{P}=0.24)$.

\section{Detached papillae in superficially invasive esophageal ad- enocarcinoma in ESD specimens}

The frequency of detached papillae in IMAC and SMAC specimens is $71.4 \%$ and $73.3 \%$, higher than BE-HGD $(0 \%, \mathrm{P}<$ 0.0001 for both). A trend of positive association of detached papillae and the presence of LVI was also noted; $57.7 \%$ of

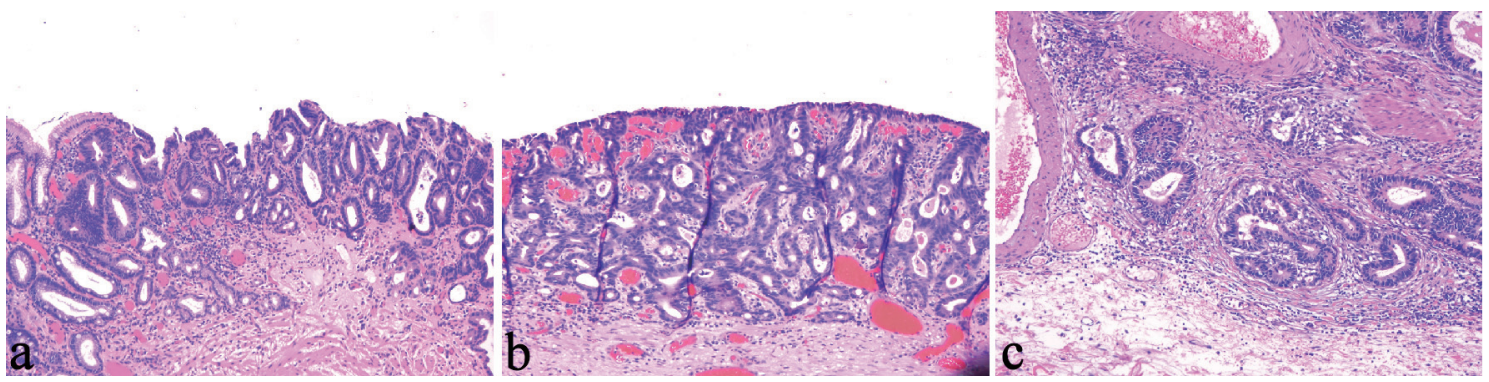

Figure 2. (a) Barrett's esophagus-related high-grade dysplasia (HGD) is characterized by confinement of pleomorphic dysplastic cells within the glandular compartment $(100 \times)$. (b) Esophageal intramucosal adenocarcinoma (IMAC) is characterized by penetration of neoplastic cells through the basement membrane $(200 \times)$. (c) Esophageal submucosally invasive adenocarcinoma (SMAC) is characterized by infiltration of neoplastic cells into the submucosa $(100 \times)$. 
Table 1. Desmoplasia in the Early Esophageal Adenocarcinoma Is Associated With Lymphovascular Invasion

\begin{tabular}{lll}
\hline & LVI N (\%) & P value \\
\hline IMAC & & \\
$\quad$ Desmoplasia $(\mathrm{n}=5)$ & $4(80)$ & 0.048 \\
$\quad$ Desmoplasia-negative $(\mathrm{n}=16)$ & $4(25)$ & \\
SMAC & & 0.24 \\
$\quad$ Desmoplasia $(\mathrm{n}=11)$ & $8(73)$ & \\
$\quad$ Desmoplasia-negative $(\mathrm{n}=4)$ & $1(25)$ & 0.006 \\
IMAC and SMAC combined & & \\
$\quad$ Desmoplasia $(\mathrm{n}=16)$ & $12(75)$ & \\
$\quad$ Desmoplasia-negative $(\mathrm{n}=20)$ & $5(25)$ & \\
\hline
\end{tabular}

IMAC and SMAC with detached papillae were positive for LVI vs. $20 \%$ of IMAC and SMAC specimens without detached papillae $(\mathrm{P}=0.06)$ (Table 2$)$.

Features associated with LVI in superficially invasive esophageal adenocarcinoma in ESD specimens identified by univariate analysis

Univariate analysis revealed that desmoplasia was associated with LVI in IMAC specimens (odds ratio (OR) 12, $\mathrm{P}=0.048$ ) and in IMAC and SMAC specimens combined (OR 9.0, P = 0.005). In addition, thickness of tumor was associated with LVI in IMAC and SMAC specimens combined (OR 2.7, P = 0.01, respectively). A trend of positive association of detached papillae and the presence of LVI was also noted in IMAC and SMAC specimens combined (OR 5.45, $\mathrm{P}=0.05$ ). Age, gender, largest dimension of ESD specimen, average thickness of resection, total tumor size, largest carcinoma size, average carcinoma size, and poor differentiation were not associated with LVI in IMAC, SMAC, or IMAC and SMAC combined (Table 3).

\section{Desmoplasia was associated with LVI by multivariable analysis}

In IMAC alone, multivariable analysis revealed that the largest dimension of ESD specimen was associated with lower frequency of LVI (OR $0.3, \mathrm{P}=0.04)$ but there was only a trend of desmoplasia predictive of LVI (OR: 479, $\mathrm{P}=0.07$ ) (Table 4). In IMAC and SMAC specimens combined, multivariate analysis confirmed that desmoplasia but not the presence of detached papillae was predictive of LVI (OR 8.0, $\mathrm{P}=0.02$; OR $4.3, \mathrm{P}=0.16$, respectively) (Table 4 ). In addition, there was a trend of thickness of tumor predictive of LVI in IMAC and SMAC specimens combined (OR 2.3, $\mathrm{P}=0.05)$ (Table 4).

\section{Discussion}

According to the current ACG guidelines, BE with HGD de-
Table 2. Detached Papillae in the Early Esophageal Adenocarcinoma Shows a Trend of Association With Lymphovascular Invasion (LVI) in IMC and SMAC Combined

\begin{tabular}{lll}
\hline & LVI N (\%) & P value \\
\hline IMAC & & \\
Presence of detached papillae $(\mathrm{n}=15)$ & $7(46.7)$ & 0.34 \\
$\quad$ Absence of detached papillae $(\mathrm{n}=6)$ & $1(16.7)$ & \\
SMAC & & 0.24 \\
$\quad$ Presence of detached papillae $(\mathrm{n}=11)$ & $8(72.3)$ & \\
Absence of detached papillae $(\mathrm{n}=4)$ & $1(25)$ & \\
IMAC and SMAC combined & & 0.06 \\
Presence of detached papillae $(\mathrm{n}=26)$ & $15(57.7)$ & \\
Absence of detached papillae $(\mathrm{n}=10)$ & $2(20)$ & \\
\hline
\end{tabular}

tected on systemic cold biopsy should undergo endoscopic eradication therapy and patients with nodularity in the BE segment should undergo EMR of the nodular lesion(s) as the initial diagnostic and therapeutic maneuver [1]. Subsequent management of IMAC and superficially SMACs partly depends on the findings on the EMR specimens. Recently, ESD has also been used to locally excise nodules in the BE segment [6]. From 2013 to 2016, a total of 44 ESD procedures were performed for BE-related neoplasm at our hospital. All specimens were en bloc resection and completely submitted for histology. This allowed us to evaluate a spectrum of features of BE-related neoplasm including desmoplasia and detached papillae, two features briefly mentioned in esophageal IMAC or studied in gastric IMAC $[5,9]$.

Our study revealed desmoplasia in $24 \%$ esophageal IMACs, similar to the $19 \%$ rate published for gastric IMACs [5]. The rate of desmoplasia in esophageal SMAC was $73 \%$ in this study. This finding supports that the diagnosis of SMAC should be based on the identifying the infiltration of neoplastic cells into the submucosa instead of relying on the presence of desmoplasia. Further, this study reveals a positive association of desmoplasia with the presence of LVI; $80 \%$ with desmoplasia were positive for LVI vs. $25 \%$ without desmoplasia (P $=0.048)$ in IMAC specimens and $75 \%$ vs. $25 \%(\mathrm{P}=0.006)$ in IMAC and SMAC specimens combined. In esophageal IMACs, the presence of LVI was associated with $27.3 \%$ of nodal metastasis $[2,8]$. Thus, IMACs with desmoplasia may theoretically at least carry $21.8 \%$ risk of nodal metastasis. Multivariable analysis revealed that desmoplasia as an independent risk factor of LVI in IMAC and SMAC specimens combined. This finding suggests that great efforts should be taken to search for LVI in IMACs and SMACs showing desmoplasia.

Our study also revealed detached papillae as a feature associated with carcinoma as it is not seen in BE-HGD. Detached papillae occur in $71.4 \%$ of esophageal IMACs and $73.3 \%$ of SMACs diagnosed in ESD specimens. A trend of positive association of detached papillae and LVI was also noted; $57.7 \%$ of IMAC and SMAC with detached papillae were positive for LVI vs. $20 \%$ of IMAC and SMAC specimens without detached papillae $(\mathrm{P}=0.06)$. These finding suggest that in ESD speci- 
Table 3. Univariate Analysis to Assess Association of Clinicodemographics, ESD Specimen Parameters, and Tumor Characteristics With Lymphovascular Invasion in Early Esophageal Adenocarcinoma

\begin{tabular}{llll}
\hline & IMAC $(\mathbf{O R}: \mathbf{9 5} \% \mathbf{C I})$ & SMAC $(\mathbf{O R : ~ 9 5 \% ~ C I ) ~}$ & IMAC and SMAC (OR: 95\% CI) \\
\hline Age & $1.09(0.98-1.2), \mathrm{P}=0.1$ & $0.88(0.73-1.1), \mathrm{P}=0.2$ & $1.03(0.96-1.1), \mathrm{P}=0.4$ \\
Gender (F/M) & $\mathrm{NA}$ & $\mathrm{NA}$ & $0.53(0.04-6.4), \mathrm{P}=0.6$ \\
Largest dimension of ESD specimen & $0.5(0.23-1.1), \mathrm{P}=0.09$ & $1.07(0.58-2), \mathrm{P}=0.8$ & $0.73(0.47-1.1), \mathrm{P}=0.2$ \\
Average thickness of resection & $1.29(0.64-2.6), \mathrm{P}=0.5$ & $\mathrm{NA}$ & $1.27(0.61-2.6), \mathrm{P}=0.5$ \\
Desmoplasia (yes/no) & $12(1.02-141), \mathrm{P}=0.048$ & $8(0.6-110), \mathrm{P}=0.1$ & $9.0(2.0-41), \mathrm{P}=0.005$ \\
Detached papillary structures (yes/no) & $4.4(0.4-47), \mathrm{P}=0.2$ & $8(0.6-110), \mathrm{P}=0.1$ & $5.45(0.96-31), \mathrm{P}=0.05$ \\
Thickness of tumor & $2.1(0.78-5.5), \mathrm{P}=0.1$ & $3.9(0.8-18), \mathrm{P}=0.09$ & $2.7(1.2-5.8), \mathrm{P}=0.01$ \\
Depth of submucosal invasion & $\mathrm{NA}$ & $\mathrm{NA}$ & $9.7(0.6-148), \mathrm{P}=0.1$ \\
Total tumor size & $1.01(0.92-1.1), \mathrm{P}=0.8$ & $1.08(0.9-1.3), \mathrm{P}=0.4$ & $1.03(0.95-1.1), \mathrm{P}=0.4$ \\
Largest size of carcinoma & $1.05(0.91-1.2), \mathrm{P}=0.5$ & $1.2(0.9-1.6), \mathrm{P}=0.2$ & $1.1(0.98-1.2), \mathrm{P}=0.1$ \\
Average size of carcinoma & $1.03(0.87-1.2), \mathrm{P}=0.7$ & $1.3(0.9-1.9), \mathrm{P}=0.2$ & $1.1(0.98-1.3), \mathrm{P}=0.1$ \\
Poor differentiation & $1.71(0.09-31.9), \mathrm{P}=0.7$ & $1.60(0.19-13.7), \mathrm{P}=0.7$ & $2.2(0.44-11.2), \mathrm{P}=0.3$ \\
\hline
\end{tabular}

OR: odds ratio; NA: not applicable.

mens with detached papillae, pathologists should look for evidence of basement membrane breaching by neoplastic glands to diagnose IMAC and search for LVI in such cases.

In line with the previous report that LVI-positive IMAC were thicker than LVI-negative IMAC [8], our study revealed tumor thickness has a trend of being associated with LVI in superficially invasive esophageal adenocarcinoma in ESD specimens. Although poor differentiation has been reported to be associated with LVI in esophageal IMAC [8], it was not associated with LVI in the current study. This may be due to a smaller number of IMAC in the current study compared to the previous study [8].

Although desmoplasia has been used as a diagnostic criterion for diagnosing SMAC in the colon [10], the mechanism leading to desmoplasia is not completely known. Studies suggested that desmoplasia, a process of fibrosis, was induced by activation of fibroblasts with increased production of extracellular matrix (ECM) proteins (collagens I, III, and XI), dysregulation of ECM turnover as a result of abnormal matrix degrading enzymes (such as matrix metalloproteinases (MMPs)), and degradation of normal type IV collagen-rich basement membrane [10-12]. Indeed, upregulation and differential expression of matrilysin (MMP-7) and metalloelastase (MMP12) occurred in BE adenocarcinoma and the expression of matrilysin was correlated with the histological aggressiveness, i.e., poor differentiation, of BE adenocarcinoma [13]. Whether MMPs present the link between desmoplasia and LVI in early $\mathrm{BE}$ adenocarcinoma remains to be determined. The mechanistic linkage between detached papillae and LVI is more elusive. However, invasive micropapillary carcinoma in gastrointestinal tract has been reported to be an aggressive variant of adenocarcinoma with high rate of LVI and nodal metastasis [14].

This study has several strengths. It is the first study to characterize the stromal changes and detached papillae in early esophageal adenocarcinoma in a cohort of ESD performed by a single endoscopist. The slides are histologically re-reviewed by a single gastrointestinal pathologist with experience in BErelated neoplasm. This study took an advantage of completely submitted ESD specimens which had better orientation and were deeper for a more accurate interpretation of early esophageal adenocarcinoma. Further, this study only included adenocarcinoma to ensure that the stromal response observed was adenocarcinoma specific.

The study harbors some limitations. The study cohort is small. Only one pathologist re-reviewed the slides and examined these two features. All study cases were from a single tertiary care center and the rate of LVI in IMAC in our study may represent an overestimate because of referral bias. This study did not correlate the biopsy diagnosis with the diagnosis in the ESD specimens as $12(27.3 \%$, out of 44$)$ were biopsied

Table 4. Multivariable Analysis to Assess if Desmoplasia and/or Detached Papillae Predict Lymphovascular Invasion in Early Esophageal Adenocarcinoma

\begin{tabular}{lll} 
& IMAC (OR: 95\% CI) & IMAC and SMAC (OR: 95\% CI) \\
\hline Desmoplasia (yes/no) & $479(0.5-422,810), P=0.07$ & $8.0(1.4-46), P=0.02$ \\
Largest dimension of ESD specimen & $0.3(0.09-0.97), P=0.04$ & NA \\
Thickness of tumor & NA & $2.3(1.0-5.4), P=0.05$ \\
Detached papillary structures (yes/no) & NA & $4.3(0.6-33), P=0.16$ \\
\hline
\end{tabular}

OR: odds ratio; NA: not applicable. 
in outside hospitals and were not available for histology rereview. Further, this study did not correlate these features with the clinical outcomes of the patients due to the relatively short follow-up and only a small number of events (death or recurrence).

In conclusion, this study revealed that desmoplasia and detached papillae can occur in IMACs. In IMACs and SMACs diagnosed on ESD specimens, the presence of desmoplasia is associated with LVI, a feature predictive of nodal metastasis in patients with early BE-related adenocarcinoma. Long-term and larger studies are needed to confirm the findings in this study.

\section{Acknowledgments}

Not applicable.

\section{Financial Disclosure or Funding}

Not applicable.

\section{Conflict of Interest}

Not applicable.

\section{Author Contributions}

All authors have contributed to the study. ZJ, DZ collected the data and drafted the manuscript; QZ and JL drafted the manuscript and critically reviewed the manuscript; AA, AE, JK, DHG, PVD contributed to the study design and critically reviewed the manuscript; $\mathrm{HX}$ analyzed the data and performed the statistical analysis; XL designed the study, interpreted the data and oversaw the project.

\section{References}

1. Shaheen NJ, Falk GW, Iyer PG, Gerson LB, American College of G. ACG clinical guideline: diagnosis and management of Barrett's esophagus. Am J Gastroenterol. 2016;111(1):30-50; quiz 51.

2. Manner H, May A, Pech O, Gossner L, Rabenstein T, Gunter E, Vieth M, et al. Early Barrett's carcinoma with "low-risk" submucosal invasion: long-term results of endoscopic resection with a curative intent. Am J Gastroenterol. 2008;103(10):2589-2597.

3. Downs-Kelly E, Mendelin JE, Bennett AE, Castilla
E, Henricks WH, Schoenfield L, Skacel M, et al. Poor interobserver agreement in the distinction of highgrade dysplasia and adenocarcinoma in pretreatment Barrett's esophagus biopsies. Am J Gastroenterol. 2008;103(9):2333-2340; quiz 2341.

4. Ormsby AH, Petras RE, Henricks WH, Rice TW, Rybicki LA, Richter JE, Goldblum JR. Observer variation in the diagnosis of superficial oesophageal adenocarcinoma. Gut. 2002;51(5):671-676.

5. Estrella JS, Hofstetter WL, Correa AM, Swisher SG, Ajani JA, Lee JH, Bhutani MS, et al. Duplicated muscularis mucosae invasion has similar risk of lymph node metastasis and recurrence-free survival as intramucosal esophageal adenocarcinoma. Am J Surg Pathol. 2011;35(7):1045-1053.

6. Yang D, Coman RM, Kahaleh M, Waxman I, Wang AY, Sethi A, Shah AR, et al. Endoscopic submucosal dissection for Barrett's early neoplasia: a multicenter study in the United States. Gastrointest Endosc. 2017;86(4):600607.

7. Lewis JT, Wang KK, Abraham SC. Muscularis mucosae duplication and the musculo-fibrous anomaly in endoscopic mucosal resections for barrett esophagus: implications for staging of adenocarcinoma. Am J Surg Pathol. 2008;32(4):566-571.

8. Li ZG, Zhu H, Shi H, Xie H, Goldblum JR, Thota PN, Liu X. Lymphovascular invasion and nodal metastasis in intramucosal adenocarcinoma of the esophagus and esophagogastric junction. J Dig Dis. 2015;16(4):197-204.

9. Lee SM, Yang S, Joo M, Kim KM, Park CK, Ahn S, Min $\mathrm{BH}$, et al. Poorly differentiated component in gastric pinch biopsies predicts submucosal invasion. Diagn Pathol. 2014;9:34.

10. Zhang D, Zhu H, Harpaz N. Overexpression of alpha1 chain of type XI collagen (COL11A1) aids in the diagnosis of invasive carcinoma in endoscopically removed malignant colorectal polyps. Pathol Res Pract. 2016;212(6):545-548.

11. Ohtani H. Pathophysiologic significance of host reactions in human cancer tissue: desmoplasia and tumor immunity. Tohoku J Exp Med. 1999;187(3):193-202.

12. De Wever O, Mareel M. Role of tissue stroma in cancer cell invasion. J Pathol. 2003;200(4):429-447.

13. Salmela MT, Karjalainen-Lindsberg ML, Puolakkainen $\mathrm{P}$, Saarialho-Kere U. Upregulation and differential expression of matrilysin (MMP-7) and metalloelastase (MMP-12) and their inhibitors TIMP-1 and TIMP-3 in Barrett's oesophageal adenocarcinoma. Br J Cancer. 2001;85(3):383-392.

14. Guzinska-Ustymowicz K, Niewiarowska K, Pryczynicz A. Invasive micropapillary carcinoma: a distinct type of adenocarcinomas in the gastrointestinal tract. World J Gastroenterol. 2014;20(16):4597-4606. 